\title{
A HISTOMORPHOLOGICAL STUDY OF BENIGN LESIONS OF ORAL CAVITY IN A TERTIARY CARE HOSPITAL
}

Harish S. Permi ${ }^{1}$, Tanu Agrawal2, Sajitha $K^{3}$, Shubha P. Bhat ${ }^{4}$, Kishan Prasad H. L ${ }^{5}$, Teerthanath Srinivas ${ }^{6}$

${ }^{1}$ Associate Professor, Department of Pathology, K. S. Hegde Medical Academy.

${ }^{2}$ Post Graduate Student, Department of Pathology, K. S. Hegde Medical Academy.

${ }^{3}$ Assistant Professor, Department of Pathology, K. S. Hegde Medical Academy.

${ }^{4}$ Assistant Professor, Department of Pathology, K. S. Hegde Medical Academy.

${ }^{5}$ Associate Professor, Department of Pathology, K. S. Hegde Medical Academy.

${ }^{6}$ Professor, Department of Pathology, K. S. Hegde Medical Academy.

\section{ABSTRACT}

The oral cavity mucosal tissue alteration can manifest in a variety of lesions where great majority of localized overgrowths of the oral mucosa are considered to be reactive rather than neoplastic in nature. Many of these lesions can be identified as specific entities on the basis of their histopathological features. In our present study, we have evaluated the various benign lesions of oral cavity with emphasis on relative frequencies, types and distribution of lesions.

\section{MATERIALS AND METHODS}

All the benign lesions of oral cavity diagnosed by histopathology in the Department of Pathology, K. S. Hegde Medical Academy of Nitte University over a period of two years from July 2012 to June 2014 were retrospectively studied. Paraffin tissue blocks were collected from Histopathology Department and 5 microns thick. Sections stained with Hematoxylin and Eosin were studied by histopathologist.

\section{RESULTS}

A total of 124 cases were analyzed during the study period. The age group ranged from 8 to 86 years in the study with a mean age of 63.9 years. Buccal mucosa was the most common site involved in 44(35.5\%) cases. Benign lesions of oral cavity included inflammatory/infective lesions in $30(24.2 \%)$ cases, traumatic/hyperplastic in $30(24.2 \%)$, cystic in $10(8 \%)$ developmental lesions in 16(13\%), pre-malignant lesions comprising of leukoplakia and erythroplakia in $20(16 \%)$ and benign neoplastic lesions constituted in 18(14.6\%) cases. Leukoplakias are the common benign lesions of the oral cavity followed by hemangiomas, hyperplasia and squamous papilloma. In the buccal mucosa, most common lesions were leukoplakia and lichen planus.

\section{CONCLUSION}

The increased frequency of benign neoplastic lesions (14.6\%) in our study and less (4\%) as compared to other author is possibly due to limited sample size in ours as contrast to large population based study by them.

\section{KEYWORDS}

Histomorphological, Benign Lesions, Oral Cavity.

HOW TO CITE THIS ARTICLE: Harish S. Permi, Tanu Agrawal, Sajitha K, Shubha P. Bhat, Kishan Prasad H. L, Teerthanath Srinivas. "A Histomorphological Study of Benign Lesions of Oral Cavity in a Tertiary Care Hospital." Journal of Evolution of Medical and Dental Sciences 2015; Vol. 4, Issue 100, December 14; Page: 16495-16498, DOI: 10.14260/jemds/2015/2451

\section{INTRODUCTION}

The oral cavity mucosal tissue alteration can manifest in a variety of lesions where great majority of localized overgrowths of the oral mucosa are considered to be reactive rather than neoplastic in nature. The manifestations of various disorders of the oral cavity clinically can be similar to the oral manifestations of certain systemic disorders making it difficult to make a correct clinical diagnosis.(1)

The extent of oral cavity extends from the skin vermillion junction of the lips to the line of circumvallate papillae below to the junction of the hard and soft palate above. It is divided into these specific areas: Mucosal lips, buccal mucosa, retromolar trigone, upper and lower alveolar ridge, hard palate, anterior two-third of the tongue and floor of the mouth.(2) A wide variety of spectrum of lesions occurs in oral cavity which includes developmental, inflammatory, cystic, reactive, hyperplastic, traumatic, premalignant and neoplastic.

Financial or Other, Competing Interest: None.

Submission 21-10-2015, Peer Review 24-10-2015,

Acceptance 07-12-2015, Published 11-12-2015.

Corresponding Author:

Dr. Harish S. Permi,

No. 203, C Block, KSHEMA Staff Quarters,

Deralakatte-575018, Mangalore.

E-mail: drharish01@gmail.com

DOI:10.14260/jemds/2015/2451
Many of these lesions can be identified as specific entities on the basis of their histopathological features and are divided into irritational fibroma, epulis fissuratum, squamous papilloma, giant cell fibroma, pyogenic granuloma and peripheral giant cell granuloma. Gingiva is common site for non-neoplastic lesions, which are usually inflammatory or represent a reaction to some kind of irritation or low-grade injury. The soft hemorrhagic lesions are highly vascular and hemorrhage is a prominent clinical and histological feature.

Pyogenic Granuloma (PG) is a tumour like growth of the oral cavity or skin that is considered to be of non-neoplastic in nature, arising commonly as a result of constant low-grade trauma, poor oral hygiene and in a few instances because of hormonal disturbances.(1) In some cases, benign lesions can be mistaken for early-stage malignant lesions leading to incorrect treatment and potentially fatal consequences for the patient.(3) In order to make a correct diagnosis, clinical characteristics of benign oral soft tissue lesions need to be well known.

The benign lesions of oral cavity tissue have a specific age, sex distribution and preferable locations. In our present study, we have evaluated the various benign lesions of oral cavity with emphasis on relative frequencies, types and distribution of lesions. 


\section{MATERIALS AND METHODS}

All the benign lesions of oral cavity diagnosed by histopathology in the Department of Pathology, K. S. Hegde Medical Academy of Nitte University over a period of two years from July 2012 to June 2014 were retrospectively studied. Demographic clinical data regarding age, sex, location and type of lesion was retrieved for each case from the biopsy register of the Medical Record Department. Paraffin tissue blocks were collected from Histopathology Department and 5 microns thick sections stained with Hematoxylin and Eosin were studied by histopathologist. The benign lesions of oral cavity were categorized and studied with respect to age, sex, location and types. Data was analyzed with the help of Chi square test to study the association between various variables. P-value less than 0.05 were considered statistically significant. SPSS 16.0 was used to carry out the analysis of data.

\section{RESULTS}

A total of 124 cases were analyzed during the study period, out of which benign lesions accounted for 33.3\%. The age group ranged from 8 to 86 years in the study with a mean age of 63.9 years. Majority of cases were seen in $5^{\text {th }}$ decade in 26 cases followed by 25 in $6^{\text {th }}$ decade, 23 in $4^{\text {th }}, 22$ in $3^{\text {rd }}, 10$ in $2^{\text {nd }}, 8$ in $7^{\text {th }}, 6$ in $8^{\text {th }}, 2$ cases each in $1^{\text {st }}$ and $9^{\text {th }}$ decades of life. (Table 1) Males were affected in 79 cases and females in 45 cases. Male:Female ratio was 1.7:1. Buccal mucosa was the most commonest site involved in $44(35.5 \%)$ cases followed by tongue in $29(23.4 \%)$, hard palate in $10(8.1 \%)$, soft palate in $3(2.4 \%)$, lip in $16(13 \%)$, retromolar trigone in $5(4 \%)$, gingiva in $3(2.4 \%)$, minor salivary gland in $3(2.4 \%)$, floor of mouth in $3(2.4 \%)$, uvula in $1(0.8 \%)$ and tonsil in $7(5.6 \%)$ cases. (Table 2).

Benign lesions of oral cavity included inflammatory/infective lesions in $30(24.2 \%)$ cases, traumatic/hyperplastic in $30(24.2 \%)$, cystic in $10(8 \%)$, developmental lesions in $16(13 \%)$, pre-malignant lesions comprising of leukoplakia and erythroplakia in $20(16 \%)$ and benign neoplastic lesions constituted in $18(14.6 \%)$ cases.

(Table 3) Inflammatory/infective lesions included lichen planus in 6 cases, pemphigus vulgaris in 1 , bullous pemphigoid in 1 , rhinoscleroma in 1 , tuberculosis 2 , leprosy in 1 , actinomycosis in 2 , rhinosporidiosis in 2 , neutrophilic dermatosis in 1 , and chronic inflammation in 13 cases. Traumatic/hyperplastic lesions were pyogenic granulomas in 8 cases, pseudoepitheliomatous hyperplasia in 10, verrucous hyperplasia in 6 , fibroepithelial polyp in 3 , and submucosal fibrosis in 3 cases. Cystic lesions were mucocele/retention cyst in 7 cases, lymphoepithelial cyst in 1, ranula (1) and keratin cyst in 1. Developmental lesions included Hemangiomas in 13 and lymphangioma in 3 cases. Premalignant lesions were leukoplakia in 19 and erythroplakia in 1 case. Benign neoplastic lesions 18 constituted squamous papilloma in 9 cases, schwannoma in 2, neurofibroma in 1 , neuroma in 1 , fibroma in 1 , angiokeratoma in 1 and pleomorphic adenoma in 3 cases.

Site wise distribution of lesions of oral cavity: In our study, $44(35.5 \%)$ lesions of buccal mucosa included tuberculosis (1), leukoplakia (12), erythroplakia (1), pseudoepitheliomatous hyperplasia (3), Verrucous hyperplasia (3), Hemangioma (5), squamous papilloma (2), neurofibroma (1), chronic inflammation (2), lymphangioma (1), submucosal fibrosis (2), lichen planus (6), pemphigus vulgaris (1), pyogenic granuloma (1), keratin cyst (1) and fibroepithelial polyp in (2) cases. Tongue in 29(23.4\%) cases which included leukoplakia in (4) cases, pseudoepitheliomatous hyperplasia in (4), verrucous hyperplasia in (1), hemangioma (4), squamous papilloma (4), chronic inflammation (4), fibroma (1), angiokeratoma (1), pyogenic granuloma (3), neuroma (1), lymphangioma (1), lymphoepithelial cyst (1). Hard palate in $10(8.1 \%)$ cases which included leukoplakia (1), pseudoepitheliomatous hyperplasia (1), pleomorphic adenoma (1), chronic inflammation (3), rhinoscleroma (1), rhinosporidiosis (1), actinomycosis (1), pyogenic granuloma (1). Soft palate in $3(2.4 \%)$ cases which included pleomorphic adenoma (1), rhinosporidiosis (1), schwannoma (1). Lip in 16(13\%) cases included verrucous hyperplasia (2), hemangioma (4), squamous papilloma (2), mucocele (6), pyogenic granuloma (2). Tonsil in $7(5.6 \%)$ cases included squamous papilloma (1), chronic inflammation (2), tuberculosis (1), schwannoma (1), lymphangioma (1), actinomycosis (1). Floor of mouth in $3(2.4 \%)$ cases included pseudoepitheliomatous hyperplasia (1), ranula (1) and mucocele (1). Retromolar trigone in $5(4 \%)$ cases included leukoplakia in (1) pseudoepitheliomatous hyperplasia (1), submucosal fibrosis (1), fibroepithelial polyp (1) and neutrophilic dermatosis in (1) cases. Gingiva in $3(2.4 \%)$ cases included leukoplakia (1), pyogenic granuloma (1), bullous pemphigoid (1). Minor salivary glands in $3(2.4 \%)$ included pleomorphic adenoma (1), chronic inflammation (2). Uvula in $1(0.8 \%)$ case was that of lepromatous leprosy (1). Leukoplakias are the common benign lesions of the oral cavity followed by hemangiomas, hyperplasia and squamous papilloma. In the buccal mucosa, most common lesions were leukoplakia and lichen planus.

\section{DISCUSSION}

A wide variety of benign soft tissue lesions either neoplastic or non-neoplastic occur in the oral cavity. The inflammatory, developmental and hyperplastic lesions which represent a reaction to injury or irritation are non-neoplastic in nature, whereas progressive autonomous growth represent neoplastic lesions. Majority of neoplastic lesions occurring in the oral cavity are benign in nature.(3) Identification of high risk population for prevention, early detection, treatment and estimation of prevalence, assessment of distribution of oral cavity lesions is possible with the retrospective studies.(4) In the oral cavity site, predilection for different types of lesions is seen. Salivary gland tumors occur most commonly on upper lip and mucocele in the lower lip. Known site predilection for different lesions will be helpful in attributing the factors responsible for the same. In our study, the age group ranged from 8 to 86 years with a mean age of 63.9 years, majority of cases seen in $5^{\text {th }}$ decade. In studies by Saraswathi et al.(5) Gambhir et al.(6) and Mathew et al.(7) age group ranged between 40-61, 20-30 and 20-40 years respectively.

Longstanding oral habits could be attributed for the occurrence of oral cavity lesions at this age group. There were only 12 and 8 cases below 20 years of age in our study and by Saraswathi et al.(5) which are inflammatory and developmental abnormalities. Buccal mucosa was the most common site in our study for the development of oral cavity lesions in 35\% cases which was similar as reported by Modi et al.(3) Ena Dowerah et al.(8) and Gambhir et al.(6) whereas it was palate in $38 \%$ cases in a study by Khateeb et al.(3)

The most common site was buccal mucosa with 32 cases (32\%) followed by lip in 22 cases (22\%).(9) Hemangiomas which were considered as developmental malformations were seen in $13(9 \%)$ cases was similar reported by Khateeb et al.(3) Majority of cases occurring in children and young adult supported that the developmental in etiology. Cystic lesions (Mucocele) are common oral lesions since their tissue of origin, i.e. salivary glands is widely distributed throughout the oral cavity. We found lower lip was the most common site. 
These findings are in agreement with previous work. Squamous papilloma was the common benign neoplasm occurred in the tongue in the 30-40 years of age group predominant in males, which was also a common benign neoplasm in males reported by Khateeb et al.(3) with peak incidence in the 11-30 years age group and most common location was palate. Among the hyperplastic/traumatic $30(24.2 \%)$ cases pseudoepitheliomatous hyperplasia in 10 and pyogenic granulomas in 8 cases were seen, where pyogenic granuloma was the most frequently encountered in $26.4 \%$ cases reported by Bashar et al.(10) Benign lesions were more common than malignant lesions. A spectrum of benign, premalignant and malignant lesions is included under Verrucous Papillary Lesions (VPLs) of oral cavity.

Majority of the benign VPLs have viral aetiology and include commonly occurring squamous papilloma along with verruca vulgaris, focal epithelial hyperplasia and condyloma.(11) In our study pseudoepitheliomatous hyperplasia, squamous papilloma and verrucous hyperplasia were seen in 10, 9 and 6 cases respectively. In our study we analysed 124 cases, out of which non-neoplastic lesions were $106(85.4 \%)$ and benign neoplastic $18(14.6 \%)$, whereas it was 36(4\%) neoplastic and 782(96\%) non-neoplastic in a study of 818 benign oral soft tissue masses by Khateeb et al.(3) Non-neoplastic lesions consisted of 336(43\%) traumatic lesions, 257(33\%) inflammatory/infective lesions, 110(14\%) cystic lesions and 73(9\%) developmental lesions. Common non-neoplastic lesions were fibroepithelial polyp, pyogenic granuloma, mucocele, hemangioma and squamous papilloma in a descending order.(3) In our study, non-neoplastic lesions constituted inflammatory/infective lesions in 30(24.2\%) cases, traumatic/hyperplastic in $30(24.2 \%)$, cystic in $10(8 \%)$ developmental lesions in $16(13 \%)$ cases. The increased frequency of benign neoplastic lesions $(14.6 \%)$ in our study and less (4\%) as compared to other author is possibly due to limited sample size in ours as contrast to large population based study by them.

\section{CONCLUSION}

The oral cavity mucosal tissue alteration can manifest in a variety of lesions where great majority of localized overgrowths of the oral mucosa are considered to be reactive rather than neoplastic in nature. Majority of benign lesions of oral cavity occurred in the $5^{\text {th }}$ decade and in males. Buccal mucosa was the most common site involved and leukoplakia was the most benign lesion encountered in our study. The increased frequency of benign neoplastic lesions $(14.6 \%)$ in our study and less (4\%) as compared to other author is possibly due to limited sample size in ours as contrast to large population based study by them.

\section{REFERENCES}

1. Altaf Hussain Chalkoo, Mirzada Bilal Ahmad. Localized benign lesions of oral cavity. A clinic ophthalmologic study of 95 cases: A retrospective analysis 2012-2014. Pakistan Oral \& Dental Journal 2015;35:179-82.

2. Modi D, Laishram RS, Sharma LD, Debnath K. Pattern of oral cavity lesions in a Tertiary Care Hospital in Manipur, India. J Med Soc 2013;27:199-202.

3. Taiseer Hussain Al-Khateeb. Benign Oral Masses in a Northern Jordanian Population-a Retrospective Study. The Open Dentistry Journal 2009;3:147-153.

4. Santosh Patil, Bharti Doni, Sneha Maheshwari. Prevalence and Distribution of Oral Mucosal Lesions in a Geriatric Indian Population. Can Geriatr J 2015;18:11-14

5. Saraswathi TR, Ranganathan K, Shanmugam S, Ramesh $\mathrm{S}$, Narasimhan PD, Gunaseelan R. Prevalence of oral lesions in relation to habits: Cross-sectional study in South India. Indian J Dent Res 2006;17:121-5.

6. Gambhir RS, Veeresha KL, Sohi R. The prevalence of oral mucosal lesions in the patients visiting a dental school in Northern India in relation to sex, site and distribution: a retrospective study. J Clin Exp Dent 2011;3:10-17.

7. Mathew AL, Pai KM, Sholapurkar AA. The prevalence of oral mucosal lesions in patients visiting a dental school in Southern India. Indian J Dent Res 2008;19:99-103.

8. Ena Dowerah, Akash Pradeep Bhuyan. Clinicopathological study of oral cavity neoplasms experience at a Tertiary Care Hospital of Assam, India. The Clarion 2014;3:1-6.

9. Mehta NV, Dave KK, Patel PS, Kadam TB. Histopathological study of oral cavity lesions: A study on 100 cases. IJCRR 2013;5:110-116.

10. Bashar A Hassawi, Eklas Ali, Nadwa Subhe. Tumors and tumor like lesions of the Oral Cavity. A Study of 303 Cases. Tikrit Medical Journal 2010;16:177-183.

11. Thomas George Kallarakkal, Anand Ramanathan, Rosnah Binti Zain. Verrucous Papillary Lesions: Dilemmas in Diagnosis and Terminology. International Journal of Dentistry 2013;2013:1-6.

\begin{tabular}{|c|c|}
\hline Age Group & $\begin{array}{c}\text { Number of Cases } \\
\text { (Total 124) }\end{array}$ \\
\hline $1-10$ & 02 \\
\hline $11-20$ & 10 \\
\hline $21-30$ & 22 \\
\hline $31-40$ & 23 \\
\hline $41-50$ & 26 \\
\hline $51-60$ & 25 \\
\hline $61-70$ & 08 \\
\hline $71-80$ & 06 \\
\hline $81-90$ & 02 \\
\hline \multicolumn{2}{|c|}{ Table 1: Showing age Distribution of no. of cases } \\
\hline
\end{tabular}

\begin{tabular}{|c|c|}
\hline Sites of Oral Cavity & No. of Cases (\%) \\
\hline Buccal mucosa & $44(35.5 \%)$ \\
\hline Tongue & $29(23.4 \%)$ \\
\hline Hard palate & $10(8.1 \%)$ \\
\hline Soft palate & $03(2.4 \%)$ \\
\hline Lip & $16(13 \%)$ \\
\hline Tonsil & $07(5.6 \%)$ \\
\hline Retromolar Trigone & $05(4 \%)$ \\
\hline Floor of mouth & $03(2.4 \%)$ \\
\hline Alveolus & $03(2.4 \%)$ \\
\hline Minor salivary glands & $03(2.4 \%)$ \\
\hline Uvula & $01(0.8 \%)$ \\
\hline Total & $124(100 \%)$ \\
\hline \multicolumn{2}{|c|}{$\begin{array}{c}\text { Table 2: Showing the sites affected and number of } \\
\text { cases of benign lesions of oral cavity }\end{array}$} \\
\hline
\end{tabular}




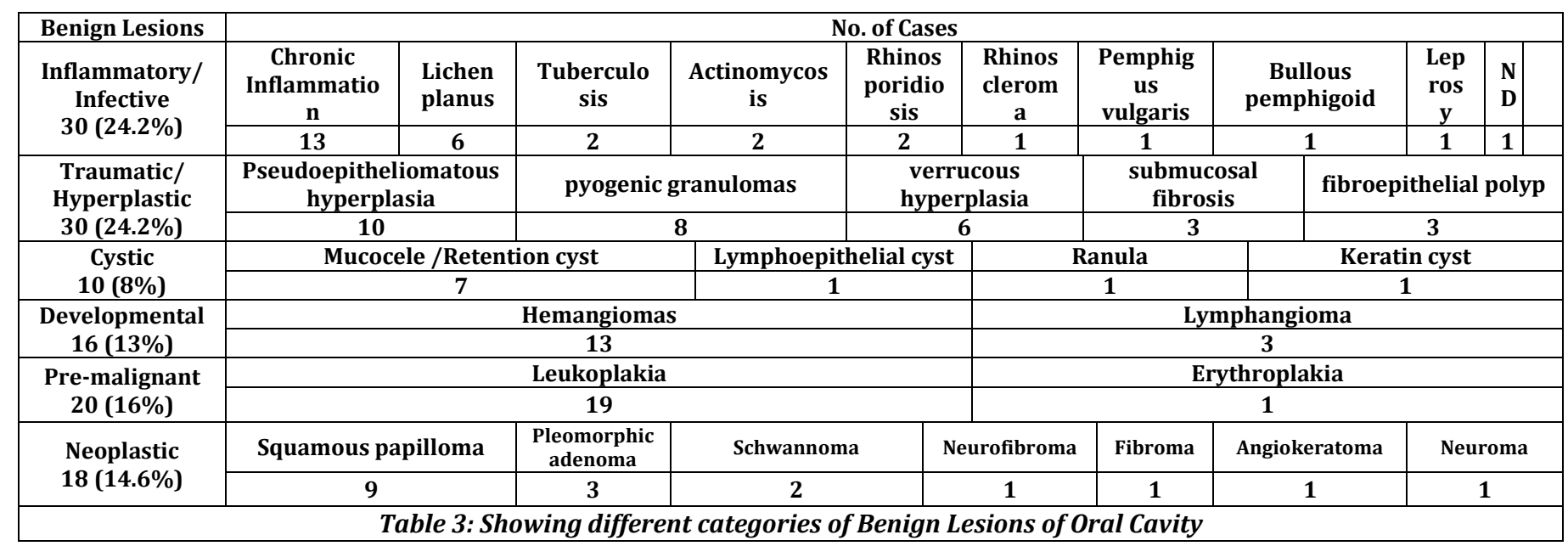

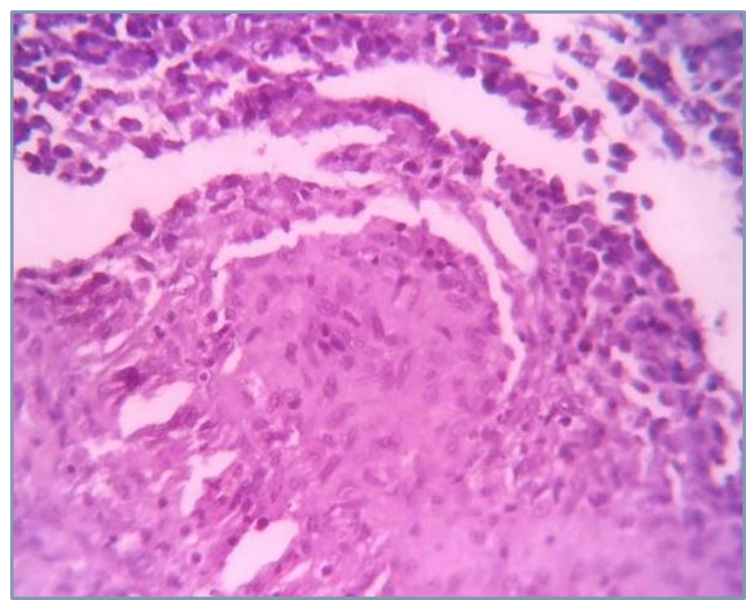

Fig. 1: Histopathology of tuberculosis tonsil showing epithelioid granulomas. (Hematoxylin \& Eosin stain, X 400)

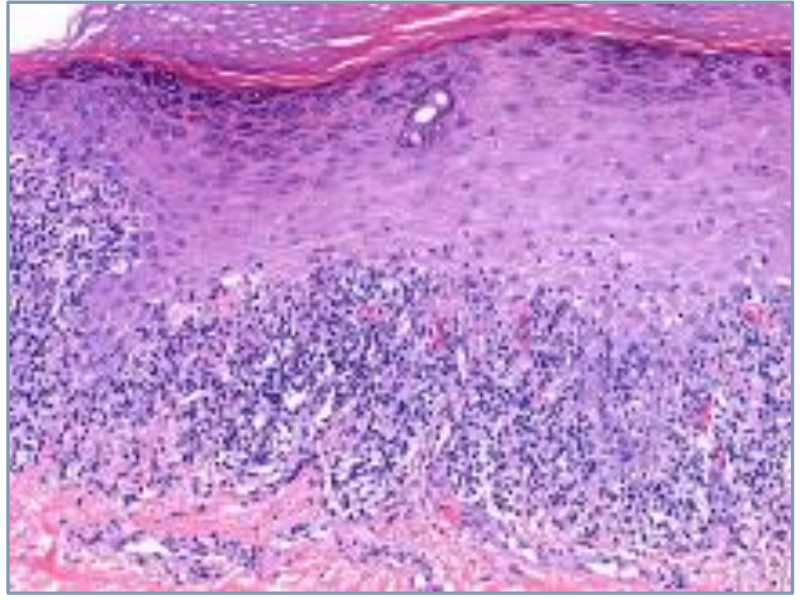

Fig. 2: Histopathology of oral lichen planus showing vacuolar degeneration of basal epithelium with band like infiltration with lymphocytes and plasma cells. (Hematoxylin \& Eosin stain, $X$ 400)

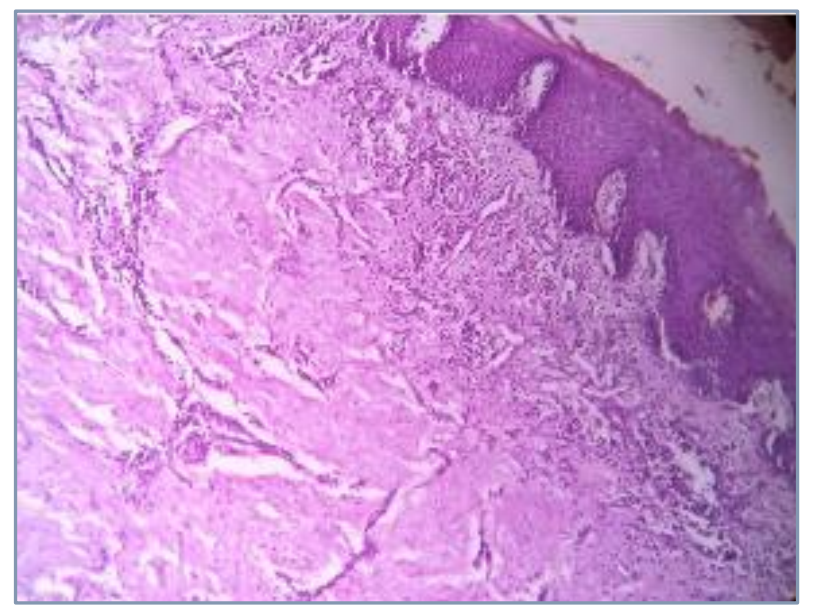

Fig. 3: Histopathology of oral submucosal fibrosis showing $f$ ibrocollagenous tissue beneath the epithelium.

(Hematoxylin \& Eosin stain, $X$ 400) 